\title{
Vibration Modal Analysis and Optimization of the Motor Base
}

\author{
Meiling Qiu ${ }^{1}$, Dafang Wang ${ }^{1,}$ a, Hui Wei ${ }^{1}$, Xiu Liang ${ }^{1}$ and Yue Ma \\ ${ }^{1}$ School of Automotive Engineering, Harbin Institute of Technology, 264209 Weihai, China
}

\begin{abstract}
When the motor works normally, its base will vibrate. The structure will generate the resonance and result in damage of the mechanical equipment when the vibration frequency reaches the natural frequency. The article which is based on the modal analysis of the motor base compares the natural frequencies in different boundary constraints in order to select appropriate way to install the motor base, and optimizes the motor base by contrasting different effects on the natural frequencies of the different thickness of the ribbed plates for selecting reasonable structure to prevent resonance.
\end{abstract}

\section{Introduction}

In recent years, all kinds of mechanical products have been developing in the direction of high precision and high quality. The performance of mechanical products has been paid more and more attention. Vibration with certain frequency and wavelength is common phenomenon. And two objects with the same vibration frequency can produce resonance so as to destroy mechanical equipment. The vibration problem is closely related to the dynamic characteristics of mechanical structure which can utilize the modal analysis to research the dynamic characteristics.

Modal is the inherent vibration characteristic of mechanical structure, which has specific natural frequency, damping ratio and mode shape. The modal analysis is helpful for the design of the motor base. The modal analysis is used to study effects on structures under different test variables by comparing and analyzing the dynamic responses [1]. Foreign research on modal analysis is earlier and more mature. They carry out the pioneering work, and establish finite element model by ANSYS software and other software in order to research the vibration characteristics [2-3]. The modal analysis is often used as a condition monitoring tool for mechanical structures such as bridges [4]. Evaluating bridge dynamic characteristics from experimental data is a common method by the modal analysis and various techniques [5]. Generally, the modal analysis is applied in engineering vibration field.

Different constraint conditions have different effects on the natural frequency. The article compares the natural frequencies of the motor base under different boundary conditions and the influence on the natural frequencies of different thickness of the ribbed plates by using the modal analysis to study the vibration characteristics of the motor base, design the structure reasonably and prevent resonance.

\section{Pre-design and modal analysis of the motor base}

\subsection{Pre-design of the motor base}

The motor base with the height of $670 \mathrm{~mm}$, the length of $980 \mathrm{~mm}$ and the width of $480 \mathrm{~mm}$ is shown in Figure 1 . There are six vertical partitions with the height of $408 \mathrm{~mm}$, the thickness of $48 \mathrm{~mm}$ and the width of $100 \mathrm{~mm}$ that are welded together. The motor base is made of structural steel, which is bolted to the ground.
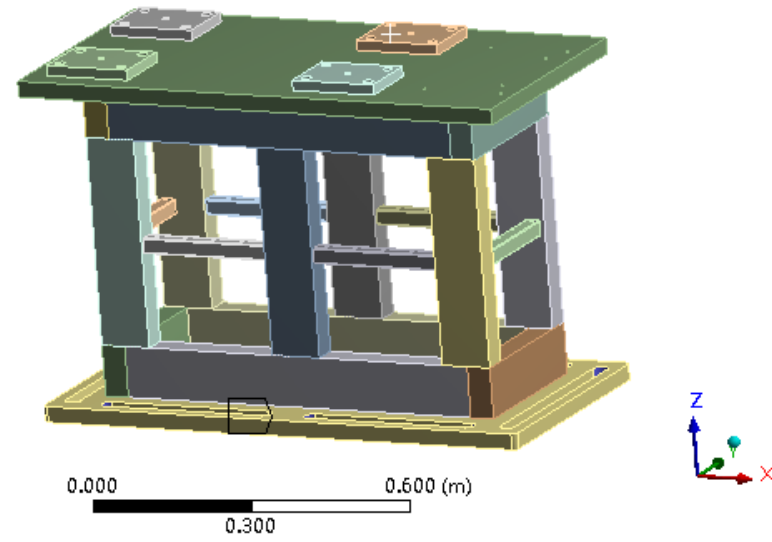

Figure 1. The structure of the motor base.

\subsection{Modal analysis of the motor base}

The general system can be described as a system with several degrees of freedom. The vibrational differential equation is

$$
M \ddot{x}+C \dot{x}+K x=F
$$

$M$ is the system's mass matrix. $C$ is the stiffness matrix and $K$ is the damping matrix. The displacement is $x . F$ is the external excitation force. Equation (1) is transformed by Laplace transformation and introduced modal coordinates in order to get the modal expansion of the

\footnotetext{
Corresponding author: wdflcjl@163.com
} 
frequency response function between the response point and the excitation point, as in

$$
H_{j p}(\omega)=\sum_{i=1}^{n} \frac{\Phi_{j i} \Phi_{p i}}{K_{i}-\omega^{2} M_{i}+j \omega C_{i}}
$$

$H(\omega)$ is the frequency response function. $\Phi$ is the mode shape matrix. The natural frequency is $\omega$.

The finite element model of the motor base is established by ANSYS software to obtain modal parameters such as natural frequency, damping ratio, and vibration mode. The motor is simplified to a rigid body because the main object is the motor base which is divided directly into meshes. The same model can be used with different meshing methods. Considering the overall structure of the motor base, it is divided by tetrahedron mesh and hexahedron mesh. Modal analysis is a pure linear analysis whose contact type is different from the contact type in nonlinear analysis. Therefore, the contact mode of the motor base is set as binding which is based on actual situation. In order to obtain better design and more accurate calculation results for the motor base. The boundary conditions are divided into three cases. Two wide sides of the motor base are fixed. Two narrow sides are fixed, and four sides are all fixed. Ultimately, the optimal layout of the motor base is determined by comparison. The lower the natural frequency is, the more easily it is to be excited. Generally, the modal effective mass of the high-order mode accounts for a very small proportion, so only the low-order mode is concerned. As to the calculation of the restraint modes of the motor base, the low-order modes are calculated. The natural frequencies with different boundary constraints are shown in Table 1

Table 1. The natural frequencies with different boundary constraints.

\begin{tabular}{|c|c|c|c|}
\hline Order & $\begin{array}{c}\text { Two wide } \\
\text { sides are } \\
\text { fixed (Hz) }\end{array}$ & $\begin{array}{c}\text { Two narrow } \\
\text { sides are } \\
\text { fixed (Hz) }\end{array}$ & $\begin{array}{c}\text { Four sides } \\
\text { are fixed } \\
\text { (Hz) }\end{array}$ \\
\hline 1 & 40.982 & 32.224 & 41.051 \\
\hline 2 & 74.067 & 66.79 & 74.706 \\
\hline 3 & 85.088 & 84.181 & 85.107 \\
\hline 4 & 244.38 & 158.45 & 244.68 \\
\hline 5 & 276.92 & 204.88 & 278.8 \\
\hline 6 & 343.35 & 296.07 & 344.99 \\
\hline
\end{tabular}

Table 1 shows that the natural frequencies are close when two wide sides and four sides are fixed. The natural frequencies are slightly smaller when two narrow sides are fixed. Due to length of space, parts of mode shapes are shown in Figure 2-4.

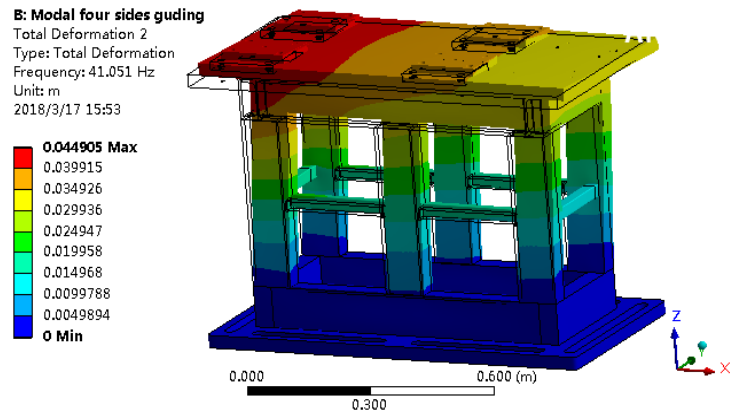

Figure 2. The first-order mode shape when four sides are fixed.

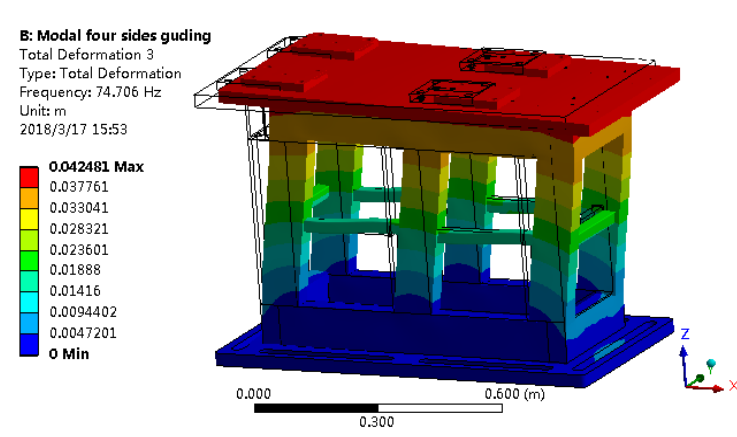

Figure 3. The second-order mode shape when four sides are fixed.

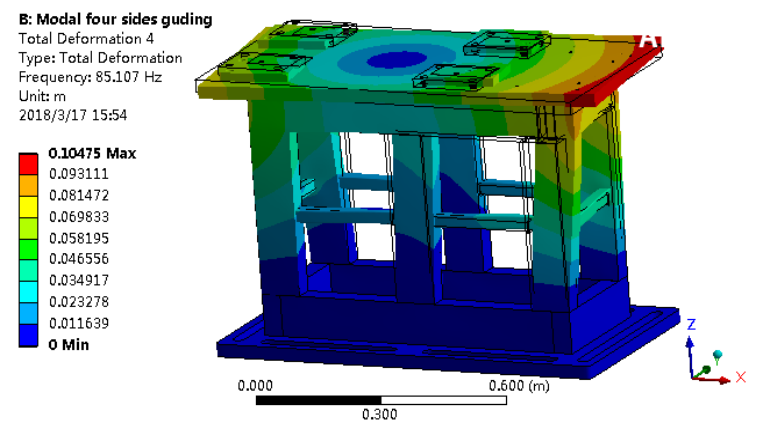

Figure 4. The third-order mode shape when four sides are fixed.

Although the boundary constraints are different, the mode shapes are basically similar except that the vibration amplitude is slightly different. The mode shape of the first-order vibrates back and forth. The mode shape of the second-order vibrates left and right, and the third-order occurs torsion. The vibration amplitude of high-order mode shapes is larger. The maximum speed of the motor is $6000 \mathrm{rpm}$ which corresponds to $100 \mathrm{~Hz}$ of the natural frequency. Resonance will happen when the frequency is lower than $100 \mathrm{~Hz}$. The low-order natural frequencies of the motor base are all lower than $100 \mathrm{~Hz}$, so it is necessary to improve the design of the motor base.

\section{Optimal design of the motor base}

The motor base need be optimized by improving the low-order natural frequency due to the resonance of the pre-design motor base. The optimization methods are strengthening stiffness and increasing the number of ribbed plates.

\subsection{The relationship between natural frequency and stiffness}

The motor base is mainly used to support the motor. The load mainly comes from the vibration that is generated when the motor works. The higher the motor speed is, the more load the motor base will bear. Therefore, the optimization of the motor base is mainly to improve the dynamic stiffness. Dynamic stiffness is excitation force required by the structure to produce a unit amplitude. Under a certain excitation force, the larger the dynamic stiffness is, the smaller the amplitude is and the stronger 
the vibration resistance is. The dynamic stiffness formula of the multi-degree-of-freedom linear system is

$$
\left[K_{\mathrm{d}}\right]=[k]-\omega_{\mathrm{a}}^{2}[m]+i \omega_{\mathrm{a}}[c]
$$

[K $\left.K_{\mathrm{d}}\right]$ is the dynamic stiffness matrix. $[k]$ is the static stiffness matrix. $\omega_{\mathrm{a}}$ is the excitation frequency. $[\mathrm{m}]$ is the mass matrix and [c] is the damping matrix. Equation (3) shows that the dynamic stiffness can be improved by improving the static stiffness matrix which can be improved by the design of the width-height ratio and increasing the number of ribbed plates. The improved motor base is shown in the Figure 5.

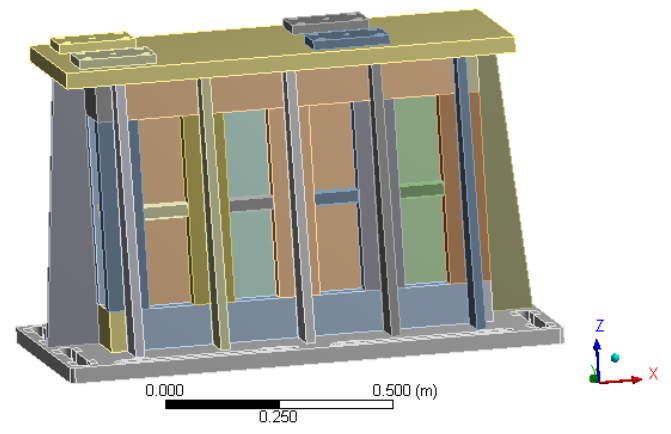

Figure 5. The improved motor base.

When the width-height ratio is closer to 1 , the overall performance of the cross section is better, which can enhance the stiffness and strength of the structure. The width is increased from $480 \mathrm{~mm}$ to $750 \mathrm{~mm}$. Also, the number of ribbed plates is increased. The motor base is calculated by using ANSYS software. The motor base is still divided by tetrahedron mesh and hexahedron mesh. The calculation time is increased due to the increased complexity of the structure. The boundary conditions are still divided into three cases. Two wide sides of the motor base are fixed. Two narrow sides are fixed, and the four sides are all fixed. The natural frequencies with different boundary constraints are shown in Table 2.

Table 2. The natural frequencies with different boundary constraints.

\begin{tabular}{|c|c|c|c|}
\hline Order & $\begin{array}{c}\text { Two wide } \\
\text { sides are } \\
\text { fixed (Hz) }\end{array}$ & $\begin{array}{c}\text { Two narrow } \\
\text { sides are } \\
\text { fixed (Hz) }\end{array}$ & $\begin{array}{c}\text { Four sides } \\
\text { are fixed } \\
\text { (Hz) }\end{array}$ \\
\hline 1 & 110.6 & 57.813 & 125.53 \\
\hline 2 & 123.43 & 151.82 & 163.19 \\
\hline 3 & 188.02 & 165.01 & 189.75 \\
\hline 4 & 313. & 255. & 393.72 \\
\hline 5 & 541.85 & 546.7 & 577.56 \\
\hline
\end{tabular}

Table 2 shows that the natural frequencies of the improved motor base are much higher than which of predesign motor base. Also, the natural frequencies are still close when two wide sides and four sides are all fixed. But the difference between the two constraints is more obvious. The natural frequencies of the improved motor base under the situation that two narrow sides are fixed, are significantly smaller than others boundary constraints. The mode shapes are similar to Figure 2-4, as shown in Figure 6-8.

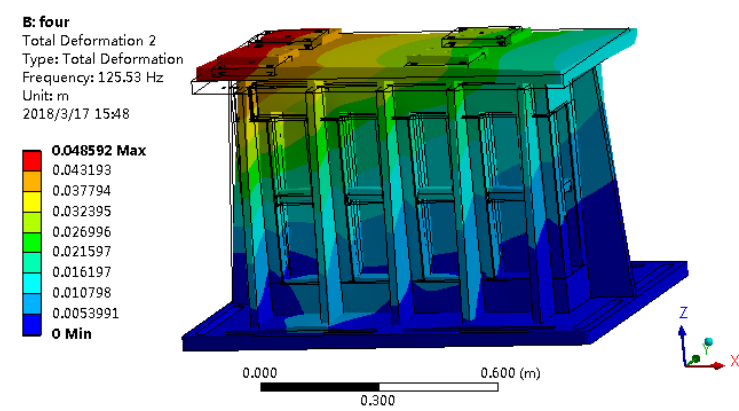

Figure 6. The first-order mode shape of the improved motor base when four sides are fixed.

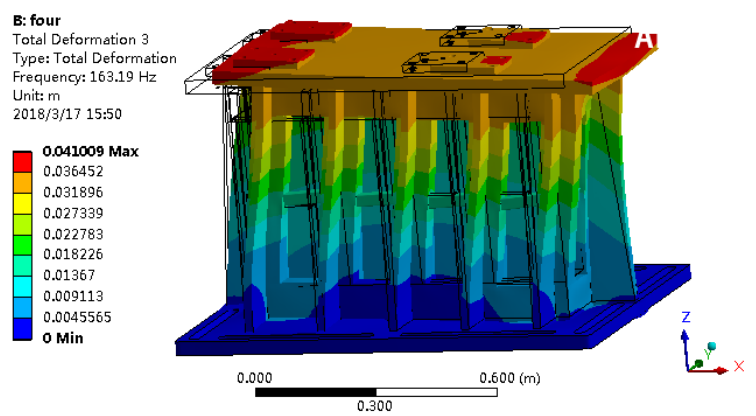

Figure 7. The second-order mode shape of the improved motor base when four sides are fixed.

The mode shape of the first-order vibrates back and forth. The mode shape of the second-order vibrates left and right, and the mode shape of the third-order occurs torsional phenomenon. Although the first-order frequency of $110.6 \mathrm{~Hz}$ under the situation that two wide sides are fixed is more than $100 \mathrm{~Hz}$, it is too close. The first-order frequency of $125.53 \mathrm{~Hz}$ under the situation that four sides are all fixed is much more than $100 \mathrm{~Hz}$, so the situation is chosen as installation method which can better prevent resonance.

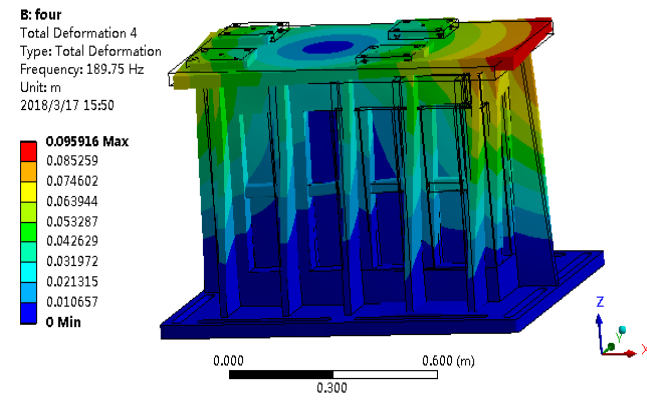

Figure 8. The third-order mode shape of the improved motor base when four sides are fixed.

\subsection{The Influence of the Ribbed Plates' thickness on natural frequency}

The maximum stiffness is not a single pursuit when optimizing structural, but economy also needs to be considered. The thickness of the ribbed plates will have influence on the stiffness, and the material of the ribbed plates is also related to economy. Therefore, the influence of the thickness of the ribbed plates on the natural frequency needs to be researched in order to select thickness of the optimal ribbed plates. The natural 
frequencies of each order under different thickness of the ribbed plates are shown in Table 3 and Figure 9.

Table 3. The natural frequencies of each order under different thickness.

\begin{tabular}{|c|c|c|c|}
\hline $\begin{array}{c}\text { Thicknes } \\
\text { s } \\
(\mathbf{m m})\end{array}$ & $\begin{array}{c}\text { First- } \\
\text { order(Hz) }\end{array}$ & $\begin{array}{c}\text { Second- } \\
\text { order(Hz) }\end{array}$ & $\begin{array}{c}\text { Third- } \\
\text { order(Hz) }\end{array}$ \\
\hline 10 & 108.68 & 151.62 & 176.61 \\
\hline 15 & 118.35 & 158.34 & 183.87 \\
\hline 20 & 125.53 & 163.19 & 189.75 \\
\hline 25 & 131.54 & 167.43 & 194.81 \\
\hline 30 & 136.78 & 171.14 & 199.39 \\
\hline
\end{tabular}

The thickness of the ribbed plates is set between $10 \mathrm{~mm}$ and $30 \mathrm{~mm}$, and the modal analysis is carried out by using ANSYS software. The larger thickness of the ribbed plates is, the higher the natural frequency is and the greater the stiffness of the motor base is. Therefore, the thickness of the ribbed plates is set to $20 \mathrm{~mm}$, which ensures that the low-order natural frequency meets the requirements and saves the material for improving the economy.

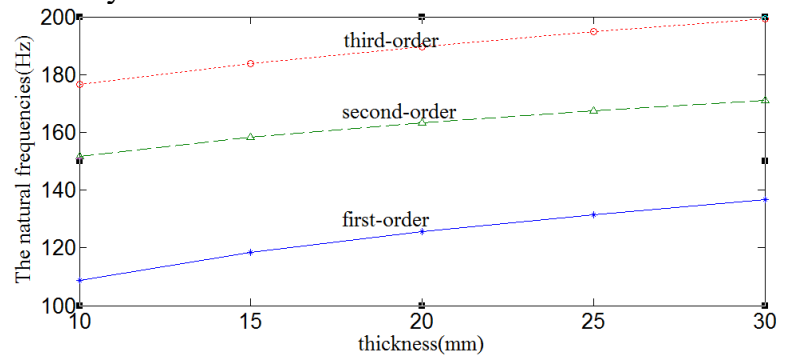

Figure 9. The natural frequencies of each order under different thickness.

\section{Conclusion}

The modal analysis has great benefit to the design of motor base. In the process of modal analysis, different constraint conditions have different effects on the natural frequency. When the four sides are all fixed, the natural frequency is the largest. The larger the thickness of the ribbed plates is, the higher the natural frequency is. But the economy should be also considered as the design factor in the design. Finally, the optimal design scheme is obtained to avoid the resonance of the motor base.

\section{Acknowledgement}

We would like to express our gratitude to the Primary Research and Development Plan of Shandong Province (2016ZDJS03A04), the Fundamental Research Funds for the Central Universities (HIT.NSRIF.201705) and the Natural Science Foundation of Shandong Province (ZR2017MEE011).

\section{References}

1. Luan J E, Tong Y T, Pek E, et al. Modal analysis and dynamic responses of board level drop test $[\mathrm{C}] / /$ Electronics Packaging Technology, 2003, Conference. IEEE, 233-243(2003).

2. AM Burke, OA Olatunbosun. New techniques in tyre modal analysis using MSC/NASTRAN[J]. International Journal of Vehicle Design, 18(2): 203212(1997).

3. Lee J 1. Vibration analysis of a vehicle body and suspension system using a substructure synthesis method $[\mathrm{J}]$. International Journal of Vehicle Design, 24(4):360-371(2000).

4. Hearn G, Testa R B. Modal Analysis for Damage Detection in Structures[J]. Journal of Structural Engineering, 117(10):3042-3063(1991).

5. Peeters B, Ventura C E. Comparative Study of Modal Analysis Techniques for Bridge Dynamic Characteristics $[\mathrm{J}]$. Mechanical Systems \& Signal Processing, 17(5): 965-988(2003). 